\title{
Comprehensive analysis of MHC class II genes in teleost fish genomes reveals dispensability of the peptide-loading DM system in a large part of vertebrates
}

\author{
Johannes M Dijkstra ${ }^{1+}$, Unni Grimholt ${ }^{2^{*} \dagger}$, Jong Leong ${ }^{3}$, Ben F Koop ${ }^{3}$ and Keiichiro Hashimoto ${ }^{1 *}$
}

\begin{abstract}
Background: Classical major histocompatibility complex (MHC) class II molecules play an essential role in presenting peptide antigens to $C D 4^{+} T$ lymphocytes in the acquired immune system. The non-classical class II DM molecule, HLA-DM in the case of humans, possesses critical function in assisting the classical MHC class II molecules for proper peptide loading and is highly conserved in tetrapod species. Although the absence of DM-like genes in teleost fish has been speculated based on the results of homology searches, it has not been definitively clear whether the DM system is truly specific for tetrapods or not. To obtain a clear answer, we comprehensively searched class II genes in representative teleost fish genomes and analyzed those genes regarding the critical functional features required for the DM system.
\end{abstract}

Results: We discovered a novel ancient class II group (DE) in teleost fish and classified teleost fish class II genes into three major groups (DA, DB and DE). Based on several criteria, we investigated the classical/non-classical nature of various class II genes and showed that only one of three groups (DA) exhibits classical-type characteristics. Analyses of predicted class II molecules revealed that the critical tryptophan residue required for a classical class II molecule in the DM system could be found only in some non-classical but not in classical-type class II molecules of teleost fish.

Conclusions: Teleost fish, a major group of vertebrates, do not possess the DM system for the classical class II peptide-loading and this sophisticated system has specially evolved in the tetrapod lineage.

Keywords: MHC, Class II, Classical, Non-classical, DM, Peptide-loading, Teleost fish, Genomics, Evolution

\section{Background}

The highly polymorphic classical MHC class II molecules can present exogenous antigenic peptides including those derived from proteins of many pathogens to $\mathrm{CD} 4^{+} \mathrm{T}$ lymphocytes in the acquired immune system $[1]$. $C D 4^{+} \mathrm{T}$ lymphocytes then can exert various functions such as helper activities toward other immune cells, e.g. B lymphocytes, macrophages and dendritic cells for their activation [1].

\footnotetext{
* Correspondence: unni.grimholt@ibv.uio.no; keihashi@fujita-hu.ac.jp ${ }^{\dagger}$ Equal contributors

${ }^{2}$ Centre for Ecology and Evolutionary Synthesis, Department of Biosciences, University of Oslo, Postboks 1066 Blindern, Oslo 0316, Norway 'Institute for Comprehensive Medical Science, Fujita Health University, Toyoake, Aichi 470-1192, Japan

Full list of author information is available at the end of the article
}

In the mammalian acquired immune system, a nonclassical MHC class II molecule, HLA-DM in humans (H2-DM in mice), plays an important role in proper peptide presentation by the classical MHC class II molecules $[1,2]$. A newly synthesized classical MHC class II molecule, which is a heterodimer composed of the $\alpha$ and $\beta$ chains, is transported from the endoplasmic reticulum to endosomal compartments including the late endosomal MIIC (MHC class II compartment) by binding to a protein called the invariant chain. The invariant chain blocks the peptide-binding groove of a classical MHC class II molecule by its "CLIP (class II-associated invariant chain peptide)" region so that other endogenous peptides cannot bind to the groove in the endoplasmic reticulum $[1,2]$.

\section{Biomed Central}

(c) 2013 Dijkstra et al.; licensee BioMed Central Ltd. This is an open access article distributed under the terms of the Creative Commons Attribution License (http://creativecommons.org/licenses/by/2.0), which permits unrestricted use, distribution, and reproduction in any medium, provided the original work is properly cited. 
After digestion of the invariant chain by endosomal proteases, CLIP is dissociated from the groove by the nonclassical class II DM molecule in the MIIC, and then other peptides, including those derived from exogenous antigens, can bind to the groove of the classical MHC class II molecule [1-6]. The DM molecule also can induce the dissociation of relatively weakly bound peptides thus showing peptide-editing function. The DM molecule itself neither exhibits classical-type polymorphism nor shows binding capacity for peptide ligands [2].

DM molecules are critical for the classical MHC class II function, as exemplified by the observation that human mutant cell lines deficient in DM molecules, and also antigen-presenting cells from the DM-knockout mice, exhibited failure in proper MHC class II peptide presentation [7-10]. Similar to a classical MHC class II molecule, a DM molecule is a heterodimer composed of $\alpha$ and $\beta$ chains, which are encoded by $D M A$ and $D M B$ genes, respectively, and possesses an overall structure similar to that of a classical MHC class II molecule, but with a unique narrow groove $[11,12]$. Orthologous $D M$ genes, $D M A$ and $D M B$, have been identified not only in many mammals but also in chickens [13] and frogs [14], indicating phylogenetic conservation throughout tetrapods. In all investigated tetrapods, the $D M$ genes reside in the $M h c$ region along with the classical $M H C$ class I and class II genes [14].

$D M$-lineage genes, however, have not been reported from the largest group of vertebrates, teleost fish, which include more than 26000 species, about $40 \%$ of all the species of vertebrates [15]. Teleost fish appear to possess effective acquired immune functions including presumable $\mathrm{T}$ lymphocyte-dependent responses to exogenous antigens [16]. Like in tetrapods, various important genes of the MHC class II system have been found in their genomes, which include genes for MHC class II $[17,18]$, CD4 $[19,20]$ and invariant chain [21-23]. The $M H C$ class II loci in teleost fish display some unique features, namely their non-linkage with $M H C$ class I loci and the lack of synteny of the class II loci between several teleost fish species [24-27]. Regarding non-classical class II genes in teleost fish, studies have been rather limited so far e.g. [28-30].

Previously, the absence of $D M$-like genes was speculated based on extensive homology searches using teleost fish genomes e.g. [14]. However, based on the negative results, one could not obtain a definitive answer, e.g., primitive DM-like molecules might be highly divergent from the known DM sequences. Very recently, the structure of a complex between a classical class II molecule and a DM molecule has been determined and the study revealed the interacting amino acid residues between the two molecules [31]. This allows us, for the first time, to analyze teleost fish class II molecules regarding the possession of the functional residues critically important for "the DM system" (in this paper defined as the peptide-loading system with the DM molecule-involved special mechanism). Taking advantage of recent progress of genome research projects, we searched and analyzed $M H C$ class II genes in teleost fish comprehensively. We typified teleost fish class II genes and their protein products based on various classical/non-classical characteristics and then examined the possession of functional residues critical for the DM system. Based on our results, we could draw a clear conclusion about the lack of the DM system in teleost fish. We also discuss possible functions of the intriguing nonclassical class II molecules revealed in teleost fish including ones newly identified in the present study.

\section{Results and discussion}

\section{Comprehensive search for teleost fish $\mathrm{MHC}$ class II genes}

Using various databases, we extensively searched for teleost $M H C$ class II genes. The ancestors of teleost fish and tetrapods have separated from each other more than 400 million years ago (Figure 1). Evolutionary relationships among relevant species are depicted in Figure 1 and also in Additional file 1: Figure S1, with more details. We identified a total of $120 \mathrm{MHC}$ class II genes or partial genes in the following Ensembl genomic databases: Danio rerio (zebrafish; ZV9), Gasterosteus aculeatus (stickleback; BROAD S1), Oryzias latipes (Medaka1), Takifugu rubripes (Fugu4.0), Tetraodon nigroviridis (Tetraodon8.0) and Oreochromis niloticus (Nile tilapia; Orenil 1.0). Seventy-eight of these sequences are devoid of apparent deletions, premature stop codons and/or frame-shifts.

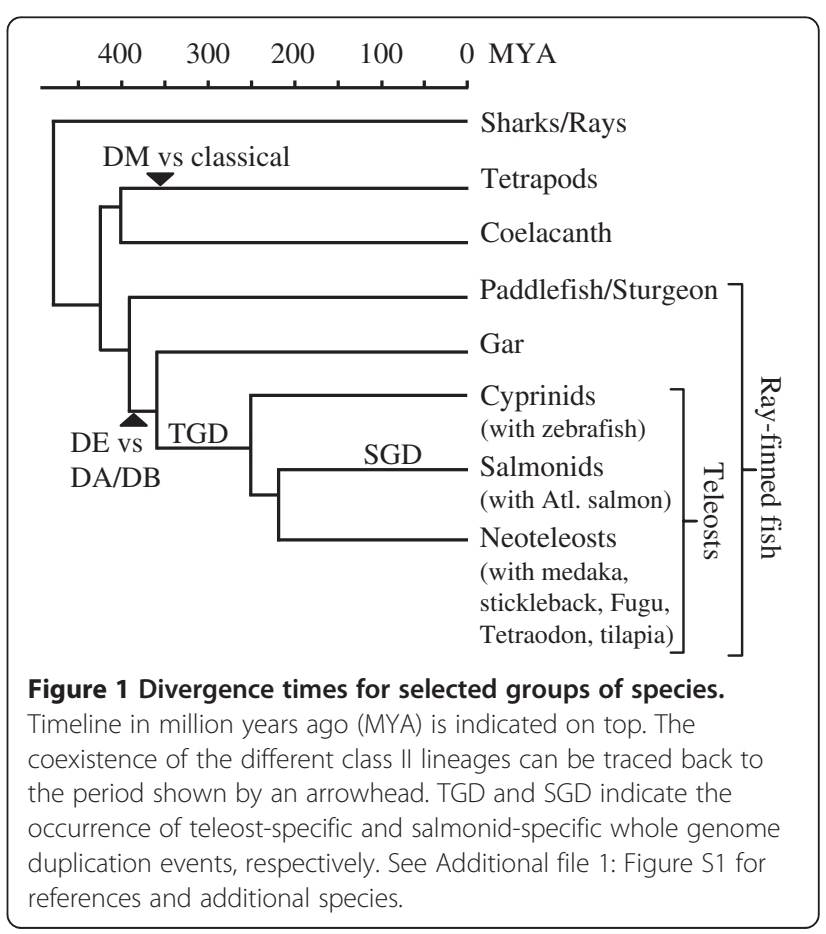


Further, we investigated our improved assembly of the Atlantic salmon genome and found five new class II genes. The $M H C$ class II sequences obtained in this study are summarized in Figure 2 (their genomic locations with surrounding genes). The amino acid sequence comparison of representative class II sequences is presented in Figure 3.

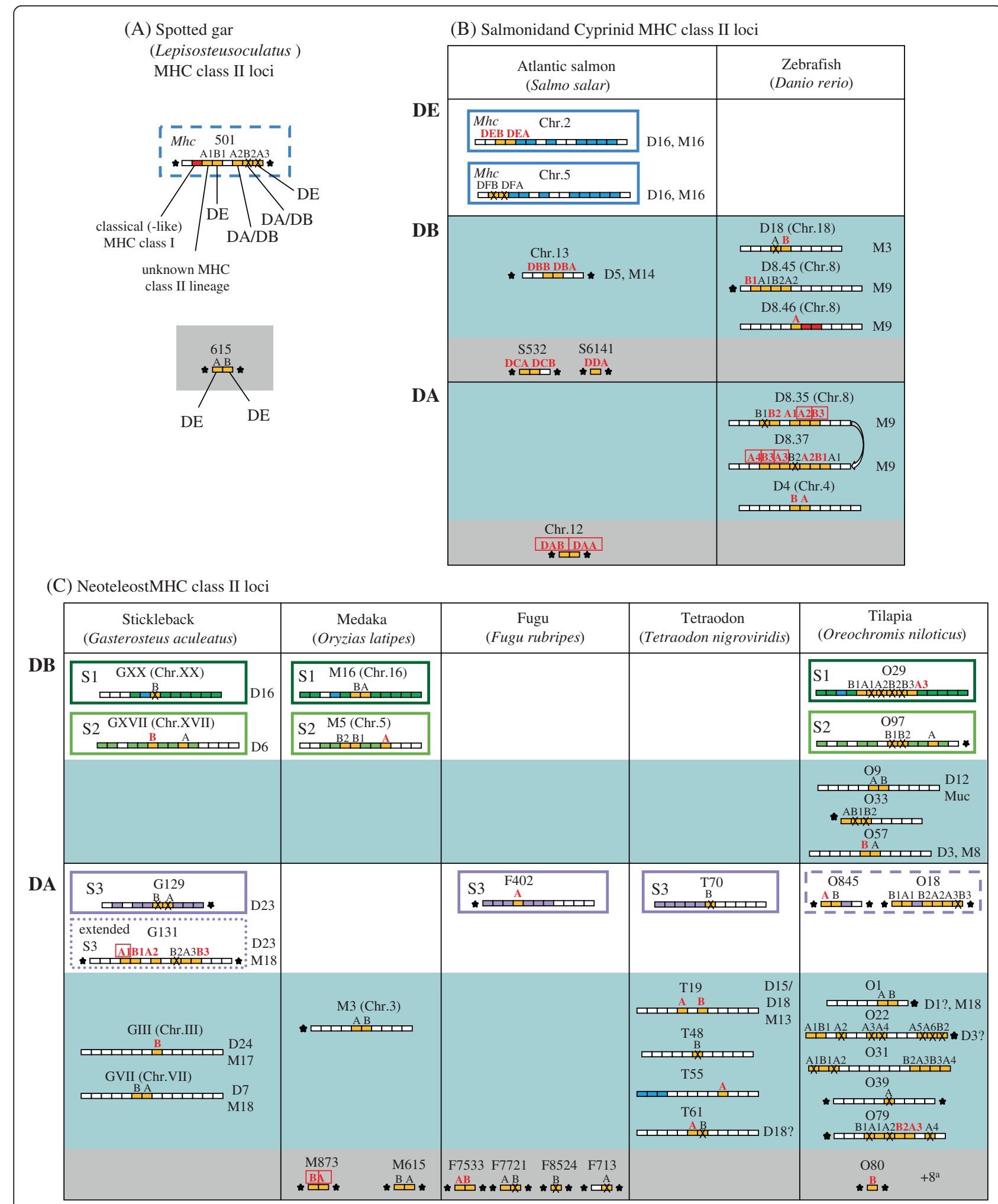

Figure 2 (See legend on next page.) 


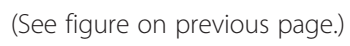

Figure 2 MHC class II loci in gar and various teleost fish. Schematic view of MHC class II A and B genomic regions as found for gar (A), salmon and zebrafish (B), and neoteleosts (C), and, for the teleost fishes, organized per DE, DB and DA group. Small blocks indicate genes, and stars indicate ends of scaffolds. Nomenclature and gene identities are explained in Additional file 2: Figure S2, and for salmon also in Additional file 8: Text S2. Synteny with class II genomic regions in other species is indicated by similar coloring of homologues: blue for Mhc-scaffold genes as found in human, dark green for neoteleost S1 region genes, lime green for neoteleost S2 region genes, and purple for neoteleost S3 region genes. Dotted and dashed purple lines indicate extended and probable S3 regions, respectively. Orange and red boxes represent MHC class II and MHC class I genes, respectively. "A" stands for a class II a chain gene and "B" for a $\beta$ chain gene. Class II A and B gene names are in red font if matching transcripts were found (Additional file 5: Table S2 and Additional file 7: Text S1), and the name is boxed if matching transcripts were abundant. Crosses indicate pseudogenes and/or genes with incomplete information. White backgrounds indicate that in syntenic regions in other species also MHC class II genes were found, blue backgrounds indicate scaffolds without such class II synteny, and gray backgrounds indicate lack of sufficient sequence information for estimation of synteny. Observed synteny between teleost regions regardless of MHC class $I$ presence (Additional file 4: Table S1) is summarized behind scaffolds by " $\mathrm{M}+$ number" and " $\mathrm{D}+$ number" for the respective chromosome numbers in medaka (M) and zebrafish (D for Danio), respectively. Linkage of classical-type class I and class II in spotted gar scaffold 501 suggests that this is an Mhc region, but more information on neighboring genes is needed (therefore dashed blue line). MHC class I genes on zebrafish Chr.8 are nonclassical [56]. The MHC class II genes and the Mhc-scaffold gene MSH5 (Additional file 2: Figure S2) in the neoteleost S1 group may or may not have derived from a direct translocation from the Mhc region on the same chromosome [33]. Tetraodon scaffold T55 has no synteny with regions in other species (Additional file 7: Table S1), so the linkage of T55 A gene with Mhc-scaffold genes (Additional file 2: Figure S2) is probably not ancestral. ${ }^{a}$ Eight tilapia regions with little informative value were omitted from the figure, but are shown in Additional file 2: Figure S2.

The phylogenetic tree analyzed based on the aligned sequences is shown in Figure 4 ( $\alpha 1$ domain of class II $\alpha$ chain) as a representative. Additional file 2: Figure S2, Additional file 3: Figure S3, Additional file 4: Table S1, Additional file 5: Table S2, Additional file 6: Table S3, Additional file 7: Text S1, Additional file 8: Text S2, Additional file 9: Text S3 and Additional file 10: Text S4 provide the detailed information.

\section{Novel expressed $M H C$ class II genes in an Mhc region of the Atlantic salmon genome and their ancient characteristics}

In a duplicated $M h c$ region on chromosome 2 (linkage group 1 (LG1); Additional file 8: Text S2) of the genome of Atlantic salmon (Salmo salar), our model fish, we could find two novel class II genes, named $D E A$ and $D E B$, the former encoding an $\alpha$ chain and the latter a $\beta$ chain of an MHC class II molecule (Figure 2B and Additional file 2: Figure S2). These genes are located closely to each other like a typical pair of MHC class II genes of mammals. A teleost fish specific whole genome duplication (Figure 1 TGD) resulted in duplication of the $M h c$ region to two different chromosomes, each experiencing subsequent rearrangements and gene losses [32,33]. Teleost classical MHC class I genes were found in only one of those two $M h c$ regions e.g. [34]. Our mapping of the Atlantic salmon $D E A / D E B$ to chromosome 2 is the first identification of intact $M H C$ class II loci within a teleost $M h c$ region (Figure 2B and Additional file 2: Figure S2), representing the TGD duplicate $M h c$ region without class I [32,33]. Early in the salmonid line an additional whole genome duplication occurred (Figure 1 SGD), which presumably resulted in duplicate $M h c$ regions on chromosomes 2 and 5 (Figure 2B and Additional file 2: Figure S2). In the Atlantic salmon $M h c$ region of chromosome 5, DE group genes also can be found, namely the pseudogenes $D F A \psi$ and
$D F B \psi$ (Figure 2B and Additional file 2: Figure S2). Both $D E A / D E B$ on chromosome 2 and $D F A \psi / D F B \psi$ on chromosome 5 are closely linked with typical $M h c$ scaffold genes such as BRD2, TAP1, PBX2 and RGL2 genes (Additional file 8: Text $S 2$ and Additional file 2: Figure S2). A possible orthologue of $D F A \psi$, equally partial and inactivated, has previously been described in the salmonid fish rainbow trout [32].

DE-related MHC class II sequences could be identified in the databases for a few other teleost fish including red seabream (a neoteleost fish), fathead minnow and dojo loach (both Cypriniformes), and importantly several primitive non-teleost ray-finned fish like spotted gar, sturgeon and paddlefish (Figures 3 and 4, Additional file 3: Figure S3, Additional file 10: Text S4). In the genome of the spotted gar (Lepisosteus oculatus; PreEnsembl Lepocu1) we could identify four DE-related sequences (Figure 2A).

In Figure 3, the predicted amino acid sequences of the membrane-distal domains of the Atlantic salmon DE molecule are compared with those of other MHC class II molecules. The DE and related sequences significantly differ from all the teleost fish class II sequences previously published. Particularly, they share several residues with class II molecules of the other jawed vertebrates (not only the classical class II molecules of tetrapods and cartilaginous fish but also the DM molecules of tetrapods) which are not observed in previously reported teleost fish class II. Those include F12, Q14, P18, R44 of class II $\alpha 1$ domain and D41 and R93 of class II $\beta 1$ domain (shaded with grey in Figure 3). Regarding specific residues of $\mathrm{DE}$ and related sequences, those consist of the following (shaded with lime green in Figure 3): R8, L10, N16, W51 of $\alpha 1$ domain and E16 of $\beta 1$ domain.

The $M h c$ regions in tetrapods and cartilaginous fish comprise a similar set of $M H C$ class I and II genes, as well as other $M h c$ scaffold genes [35,36]. In tetrapods, 


\begin{tabular}{|c|c|}
\hline 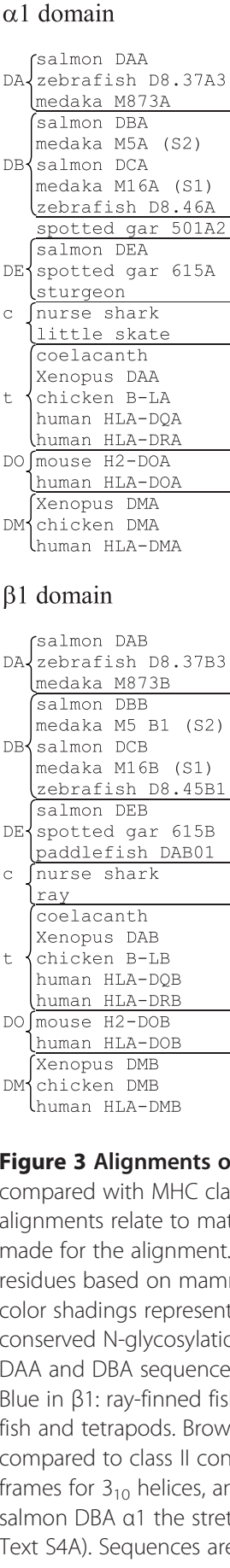 & 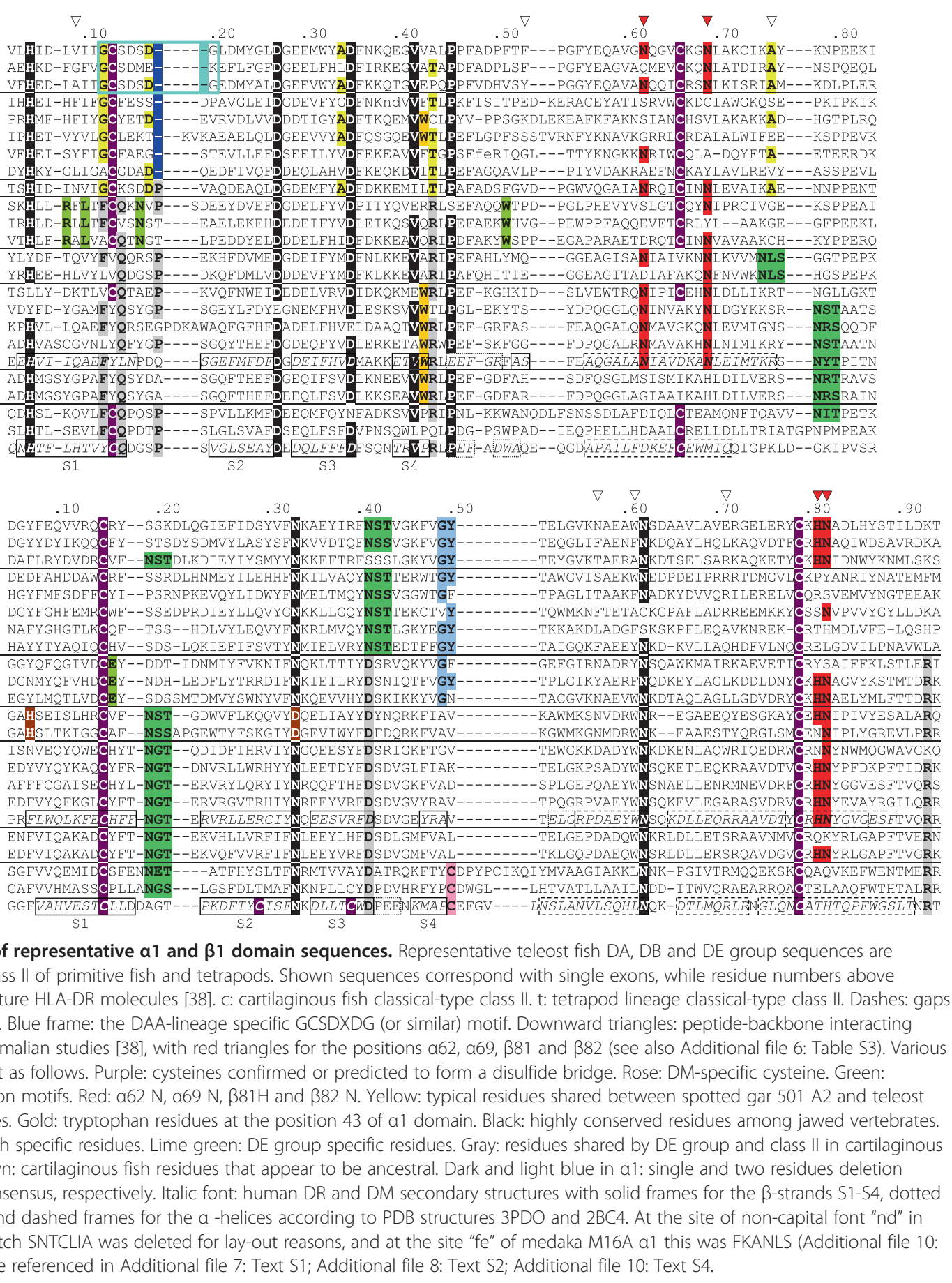 \\
\hline
\end{tabular}

the $D M$ genes also reside in this region. The teleost fish genomes, in contrast, represent a derived situation in that the classical $M H C$ class I and II genes are not linked [24,26]. In the primitive ray-finned fish spotted gar genome, we found that a classical-type class I gene (for its molecular features, see Additional file 2: Figure S2) is linked with class II genes (Figure 2A), which suggests that the non-linkage between classical class I and II was established only within teleost fish.

\section{Clarification of the three major MHC class II groups in teleost fish}

With the addition of a newly identified group, teleost fish class II genes can be organized into three major groups, 


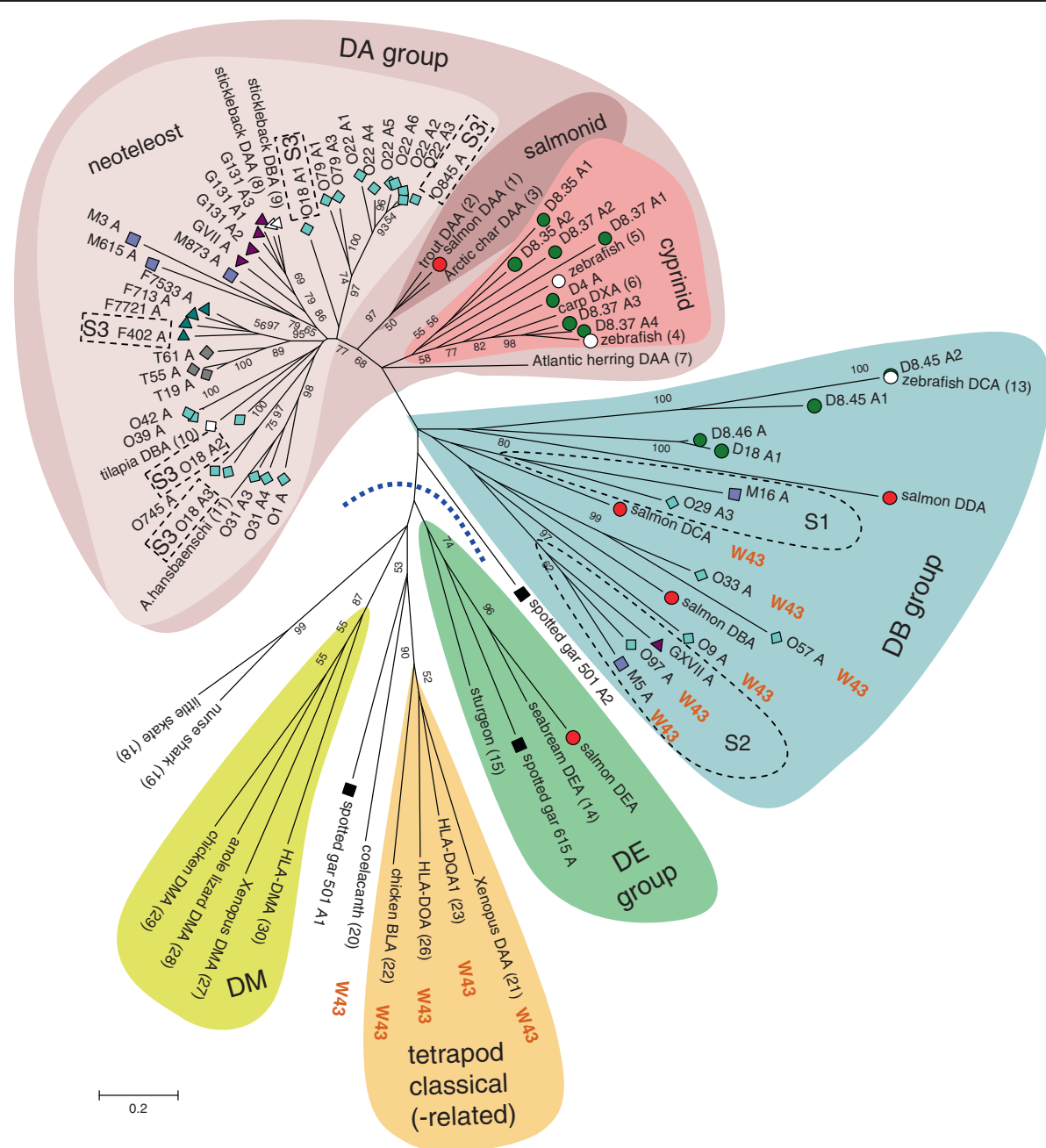

Figure 4 Phylogenetic tree of MHC class II a1 domain sequences. The sequences which possess aW43 are labeled with the orange letter "W". The bootstrap consensus tree was inferred from 500 replicates using the Neighbor-Joining method [61]. The tree is drawn to scale, with branch lengths representing the number of amino acid substitutions per site (see scale bar). Numbers behind names relate to references listed in Additional file 10: Text S4. Sequences that are also depicted in Figure 2 have their names with colored shapes indicative for the species: black square for spotted gar, red circle for Atlantic salmon, green circle for zebrafish, purple square for medaka, violet triangle for stickleback, gray diamond for Tetraodon, teal triangle for Fugu, blue diamond for Nile tilapia. Identical shapes, but white, are used for other reported sequences of those species. DM sequences are shown with the yellow background color. Tetrapod classical (-related) sequences are shown with the orange background color. The other background colors distinguish DA, DB and DE sequences, and within the DA group also cyprinid, salmonid and neoteleost sequences are distinguished. The dashed blue line divides the DA/DB lineage from the other class II sequences. S1, S2 and S3 indications plus dashed lines refer to products of genes situated in the respective synteny regions (Figure 2). Spotted gar 501A1 shows some interesting similarity with MHC class II of other classes of vertebrates (see also Additional file 10: Text S4 and Additional file 3: Figure S3B), but this single sequence does not provide sufficient information for valid discussion.

namely, DA, DB (named after a zebrafish DB sequence) and DE group, based on several sequence features including specific insertions and/or deletions (Figure 3 and Additional file 10: Text S4). Previously, reported DA and DB group sequences were classified as classical and nonclassical class II, respectively, assignments which were based only on polymorphism and expression analyses and not on comparison of characteristic amino acid residues (Additional file 9: Text S3). The DE and DA groups are well supported as distinct lineages by phylogenetic tree analyses. And the tree shows that early in ray-finned fish evolution there was a separation between DE lineage and DA/DB lineage, and that from the latter the DA lineage sprouted in teleost fish (Figure 4 and Additional file 3: Figure S3). How the DA lineage relates to extant lineages within the teleost DB group is not clear at present. Three discernible lineages within the DB group are represented by (i) genes found in zebrafish, (ii) the Atlantic salmon $D C A / D C B$ plus genes found in the neoteleost genomic synteny region "S1", and (iii) the Atlantic salmon $D B A$ / 
$D B B$ plus genes found in the neoteleost genomic synteny region "S2" (Figures 2, 3, 4 and Additional file 4: Table S1).

Previous studies on teleost fish DA and DB group genes reported a lack of synteny between teleost fish class II genomic regions, suggesting the occurrence of multiple translocations and locus turnovers e.g. [25,27]. Our study largely agrees with and extends those previous observations in these respects (Figure 2 and Additional file 4: Table S1), although within neoteleosts, some syntenic MHC class II regions could be found (Figure 2: Additional file 1: Figure S1, Additional file 2: Figure S2 and Additional file 3: Figure S3). The observation that the classical DA lineage shows little locus conservation is unexpected since the longevity of a locus should be helpful for the maintenance of allelic variations. For example, a high rate of loci turnover was claimed to be responsible for the limited diversity of the MHC class I genes in the cotton-top tamarin [37] and, further, polymorphic MHC class I genes tend to map to more ancient genomic regions than non-polymorphic ones throughout the jawed vertebrates e.g. [34].

\section{Classical and non-classical features of teleost fish class II genes}

To compare with tetrapod classical and non-classical class II genes, various features of teleost fish class II genes are investigated in the following and summarized in Table 1. In short, the DA group contains classical molecules whereas the DB and DE groups comprise non-classical molecules. It is intriguing that some teleost fish nonclassical class II share the listed features with non-classical class II in tetrapods, since the ancestors of teleost fish and tetrapods separated from each other more than 400 million years ago (Figure 1). Characteristic classical and non- classical class II features thus have coexisted for a very long evolutionary time.

\section{Expression and polymorphism}

The DA group includes all the highly expressed teleost class II genes (Figure 2, Additional file 5: Table S2) and previously reported polymorphic ones (Additional file 9: Text S3). There have been relatively few reports on teleost non-classical class II genes e.g. [28-30] (Additional file 9: Text S3), and our present study added identification, gene-specific expression and polymorphism analyses of the Atlantic salmon $D C B$ (DB group), $D E A$ and $D E B$ (DE group) (Additional file 8: Text S2). Together with our previous analyses of Atlantic salmon DB group genes, $D B A, D B B, D C A$ and $D D A$ [29], and non-specific transcriptome analysis in the present study (Additional file 8: Text S2), the results show that all these genes are essentially non-polymorphic and expressed at much lower levels than the classical $D A A$ and $D A B$ genes of the DA group in the same species. Especially the expression of Atlantic salmon $D E A$ and $D E B$ is very low. The Atlantic salmon genes $D C A, D C B$ and $D E A$ have rather tissue-specific expression patterns unknown for mammalian class II genes (Additional file 8: Text S2).

\section{Peptide binding capacity}

Only DA molecules, but not DB and DE, display a high degree of conservation of the $\alpha 1$ domain residues $\alpha$ N62 and $\alpha \mathrm{N} 69$ and the $\beta 1$ domain residues $\beta \mathrm{H} 81$ and $\beta \mathrm{N} 82$, which in mammalian classical molecules make important hydrogen bonds with the backbone of peptide ligands [38,39] (Figure 3, Table 1, Additional file 10: Text S4 and Additional file 6: Table S3). Best conserved is $\beta$ N82, known

Table 1 Comparison of various features of class II genes and their protein products in jawed vertebrates

\begin{tabular}{|c|c|c|c|c|c|c|c|c|c|}
\hline & $\begin{array}{l}\text { Cartilaginous fish } \\
\text { classical }\end{array}$ & $\begin{array}{c}\text { Gar DA/ } \\
\text { DB }\end{array}$ & $\begin{array}{l}\text { Gar } \\
\text { DE }\end{array}$ & $\begin{array}{l}\text { Teleost } \\
\text { fish DA }\end{array}$ & $\begin{array}{l}\text { Teleost } \\
\text { fish DB }\end{array}$ & $\begin{array}{l}\text { Teleost } \\
\text { fish DE }\end{array}$ & $\begin{array}{l}\text { Tetrapods } \\
\text { classical }\end{array}$ & $\begin{array}{c}\text { Mammals } \\
\text { DO }\end{array}$ & $\begin{array}{c}\text { Tetrapods } \\
\text { DM }\end{array}$ \\
\hline Located at Mhc & $++^{a}$ & $(+)^{b}$ & $(+)^{b}$ & - & - & + & + & + & + \\
\hline $\begin{array}{l}\text { Linked with classical- } \\
\text { type class I }\end{array}$ & $+{ }^{a}$ & + & + & - & - & - & + & + & + \\
\hline $\begin{array}{l}\text { Classical-type } \\
\text { polymorphism }\end{array}$ & $+{ }^{a}$ & & & + & - & - & + & - & - \\
\hline Expression & $++^{a}$ & & & high & low-med & low & high & med & med \\
\hline $\begin{array}{l}\text { Peptide-binding } \\
\text { residues }{ }^{c}\end{array}$ & + & $(+)^{d}$ & $(+)$ & + & $(-)$ & $(-)$ & + & $(-)$ & - \\
\hline $\begin{array}{c}\text { CD4-binding } \beta S 144, \\
\beta E 162\end{array}$ & - & & + & + & $+1-{ }^{e}$ & + & + & + & - \\
\hline $\begin{array}{l}\text { Endosomal sorting } \\
\text { motif }^{\mathrm{f}}\end{array}$ & - & & & - & $+1-e$ & - & - & - & + \\
\hline aW43 & - & - & - & - & $+1-{ }^{e}$ & - & + & + & - \\
\hline Amino acid at a125 & G & $\mathrm{N}$ & $D$ & G & HNDKG & $D$ & NK & ND & $\mathrm{N}$ \\
\hline
\end{tabular}

Features of class II genes and those of their protein products are compared in the upper four and in the lower five rows, respectively. Details for expression and polymorphism are described in Additional file 8: Text S2 and Additional file 9: Text S3. Sequence comparisons are based on Figure 3, Additional file 10: Text S4 and ref. 31. Blanks indicate no information. ${ }^{a}$ Based on ref. 35 and references cited therein; ${ }^{b}$ Probable $M h c ;{ }^{c}$ aN62, aN69, $\beta$ H81, and $\beta N 82$. Pluses show relatively high conservation, and parentheses indicate partial situations; ${ }^{d}$ No information for the $\beta$ chain; ${ }^{e}$ Some possess these features and some do not; ${ }^{f}$ Tyrosine-based. 
to be of particular importance [40] (Additional file 6: Table S3). At the other three positions, teleost fish DA molecules exhibit some variations comparable to some species in mammals (Additional file 6: Table S3). Although the presence of such peptide-binding residues in DA molecules has been reported before [41], the detailed analyses of teleost fish DB molecules have not been reported. Among the DA group, a few DA molecules lack these peptide-binding residues and might exert nonclassical functions akin to DO in mammals, which diverged from classical molecules in relatively recent times.

\section{CD4 binding capacity}

Recently, several amino acid residues important for the interaction between CD4 and class II molecules were revealed [42]. Two of those residues at the interface of the two molecules appear highly conserved throughout tetrapod species, namely $\beta S 144$ and $\beta E 162$ (Additional file 10: Text S4). We found that these two residues are also highly conserved in teleost fish DA, whereas cartilaginous fish classical molecules, tetrapod DM molecules and some teleost fish DB molecules lack these residues (Table 1 and Additional file 10: Text S4).

\section{Endosomal sorting motif}

A characteristic feature of the non-classical DM molecules is the possession of an endosomal sorting motif in the $\beta$ chain cytoplasmic tail $[43,44]$ (Table 1 ). We found potential tyrosine-based endosomal sorting motifs in the $\beta$ chain of a few teleost DB group molecules although their location differs from those in the DM molecules (Table 1 and Additional file 10: Text S4E).

\section{Preservation of classical genes throughout teleost fish}

Based on the current database information, all the teleost fish species that we investigated have DA, most of them have $\mathrm{DB}$, and a few of them have $\mathrm{DE}$ group genes. Conservation of gene copies preserving classical features accompanied by seemingly random loss of older nonclassical gene duplicates is somewhat reminiscent of MHC class I evolution described for higher vertebrates [45]. However, the mode of the teleost fish MHC class II evolution highly contrasts with that of the tetrapod species in which not only the classical class II but also the non-classical $D M$ genes are highly conserved. Recently, it was reported that Atlantic cod, a teleost fish, does not possess various genes in the MHC class II system such as those for MHC class II molecules, CD4 and invariant chain [27]. Although this apparent loss of the MHC class II system should have various disadvantages and actually the cods are known to have a poor adaptive antibody response, they do survive and thrive. The cod situation reflects the plasticity of the teleost fish immune system in which other factors may adapt to a large variation in the MHC class II system [27].

\section{Phylogeny of the DM system through the window of the critical functional residues}

A critical residue of the DM system is not found in the teleost fish classical class II molecules

Recently, the structure of HLA-DM/HLA-DR1 complex was clarified [31]. The overall structure of the complex is largely consistent with the previous independent estimation of the interface of the two molecules based on experiments using mutagenesis [46-49] and tethered complexes [50]. In the side-by-side structure, the interface is mainly formed by the $\alpha$ chains of the two molecules, and a lateral surface of the DR $\alpha 1$ domain, close to the N-terminus of the peptide-binding groove, interacts with $\mathrm{DM} \alpha$ chain and additionally DM $\beta 1$ [31]. The structural study revealed two key amino acid residues ( $\alpha \mathrm{N} 125$ of DM and $\alpha \mathrm{W} 43$ of DR1) in the interaction between the DM molecule and the classical class II DR1 molecule at $\mathrm{pH} 5.5$, which is within the range of the physiological late endosomal $\mathrm{pH}$ suitable for the $\mathrm{DM}$ activity [31]. In the structure of the DM/DR1 complex, a tryptophan residue of the DR1 molecule ( $\alpha$ W43) flips from the original location and its indole ring nitrogen atom interacts with an asparagine of the DM molecule $(\alpha \mathrm{N} 125)$ through a hydrogen bond [31]. This was elucidated by comparison between the structures of the DM-bound [31] and -unbound DR molecule [51]. The change of $\alpha \mathrm{W} 43$ position is accompanied by conformational alterations in the P1 pocket peptide-binding region of the DR1 molecule, which include the novel formation of a long $\alpha$-helical segment with a short break and the repositioning of the hydrophobic $\alpha \mathrm{F} 51$ into the P1 pocket [31]. These changes explain the dissociation of CLIP, the stabilization of empty class II molecules, and further the selection of high affinity peptide ligands [31]. The previous study indicated that $\alpha \mathrm{W} 43 \mathrm{~F}$ mutation of the DR molecule greatly reduced both the DM function and the binding to DM molecule [48]. The effects of $\alpha \mathrm{N} 125 \mathrm{~A}$ and $\alpha \mathrm{N} 125 \mathrm{R}$ mutations in the DM molecule were also examined and these mutations caused a loss of both the DM activity and the binding to the DR molecule [31]. HLA-DO, a human non-classical class II molecule, which can bind tightly to the DM molecule and is known to be an inhibitory modulator of the DM molecule, also possesses $\alpha \mathrm{W} 43$. The structure of HLA-DM/ HLA-DO complex was independently reported very recently and it also revealed the important participation of $\alpha W 43$ of HLA-DO and $\alpha$ N125 of HLA-DM in the complex formation in which HLA-DO behaves as a mimic of the classical class II molecule [52]. Very importantly, the classical class II $\alpha$ chains of the DA group of teleost fish do not possess the $\alpha \mathrm{W} 43$ critical for the interaction with 
the DM molecule (Table 1, Figure 4, Additional file 10: Text S4A).

As $\alpha \mathrm{W} 43$ constitutes a part of the $\beta$-strand 4 and there are a few highly conserved amino acid residues near this position, we did not have any difficulties with the alignment of the position 43 between teleost fish and tetrapod class II $\alpha$ chain sequences. At the amino acid position corresponding to $\alpha \mathrm{W} 43$, the teleost fish classical-type class II molecules, namely, those belonging to the DA group, exhibit variability (Additional file 10: Text S4A), but no tryptophan residue was observed. Retrospectively, the absence of a tryptophan and also some variability at the position 43 of the class II $\alpha$ chain of teleost fish can be recognized in a previous study using a few teleost fish sequences [41] and also in the other studies when the sequence alignments are adjusted e.g. $[29,53]$. However, the meaning of these observations could not be understood in relation to the DM system before. In the present study, we comprehensively investigated various teleost fish genomes and examined many DA group members. We did not find any sub-lineages in the DA group in which class II $\alpha$ chains specifically possess $\alpha \mathrm{W} 43$ (Additional file 10: Text S4A). The variable nature of the position 43 of the class II $\alpha$ chain without specific conserved residues suggests that the teleost fish classical class II molecules do not use this amino acid position for the interaction with some regulatory molecules. Rather, teleost fish appear to use this position to further increase the variation of the pocket.

\section{Teleost fish aW43-containing class II molecules are classified as non-classical}

In the DB group of teleost fish, we could find that six sequences (M5A of medaka, O97A, O9A, O57A and O33A of tilapia and DCA of Atlantic salmon) possess $\alpha \mathrm{W} 43$ (Additional file 10: Text S4A). Although our previous study reported that the Atlantic salmon $D C A$ gene for the $\alpha$ chain was not polymorphic based on the EST information [29], the identification and analysis of $D C B$ gene for the $\beta$ chain in the present study was important, as we know examples in which the extent of polymorphism is highly different between the genes coding for the $\alpha$ and $\beta$ chains. In the case of human classical class II $D R$ genes, $D R A$ shows only limited polymorphism while $D R B$ shows very high polymorphism [54]. In the present study, the low polymorphism of $D C B$ gene was clarified, and therefore we could classify the plausible pair of the Atlantic salmon DCA/DCB molecule as non-classical with the other supporting observations described in the following. Consistent with the non-classical feature of lacking polymorphism, DCA lacks both peptide-binding asparagine residues $(\alpha \mathrm{N} 62$ and $\alpha \mathrm{N} 69)$ although DCB retains $\beta$ N82. For the position 69, DCA possesses a hydrophobic residue like the DM sequences, a feature shared with most of the other teleost fish DB sequences (Additional file 6:
Table S3). Further, both $D C A$ and $D C B$ genes showed unique expression patterns. They are expressed predominantly in the digestive tract, as observed both in the RTPCR study and in the transcriptome analysis (Additional file 8: Text $\mathrm{S} 2$ ). These expression patterns are quite different from those of the classical class II genes of both teleost fish DA group and tetrapods. The classical MHC class II genes of human and Atlantic salmon show their highest expression in various immunologically important tissues when investigated with transcriptome analyses, and also human $D M$ genes, HLA-DMA and HLA-DMB, show similar expression patterns (Additional file 8: Text S2). Another characteristic of DCB is that, as already mentioned briefly, DCB interestingly possesses a putative tyrosinebased endosomal sorting motif in its cytoplasmic tail like the DM molecules, although the position of this motif is different from those observed in the DM molecules (Additional file 10: Text S4E). This endosomal sorting motif can also be observed in molecules relatively closely related to DCB (Additional file 10: Text S4E). Nonclassical identity of DCB could not be deduced from the analysis of the CD4-binding residues as DCB possesses both $\beta S 144$ and $\beta E 162$ (Additional file 10: Text S4D), but the retention of these residues in non-classical molecules can also be observed in other cases such as HLA-DO (Additional file 10: Text S4D).

Similar to DCA, the other five teleost fish class II $\alpha$ chain sequences that possess $\alpha \mathrm{W} 43$ could be classified as non-classical based on the analysis of the residues important for peptide-binding although we do not have clear information about their polymorphism and expression patterns (Additional file 10: Text S4). Their apparently intact, presumable $\beta$ chain partners (three out of five) could also be classified as non-classical as they lack the important peptide-binding residues (both $\beta \mathrm{H} 81$ and $\beta \mathrm{N} 82$ in two cases and $\beta \mathrm{H} 81$ in one case) and also lack at least one of the CD4-binding residues (Additional file 10: Text S4). All the identified teleost fish class II $\alpha$ chains that contain $\alpha \mathrm{W} 43$ belong to the $\mathrm{DB}$ group of the synteny region "S1" or "S2" of neoteleost fish or their closely related molecules including the Atlantic salmon DCA and together they seem to have descended from a common ancestral molecule in the early phase of the evolution of the DB group (Figure 4).

In addition to $\alpha \mathrm{W} 43$, the residues $\alpha \mathrm{K} 38$ and $\alpha \mathrm{E} 40$ of the DR molecule also participate in the formation of the hydrogen bonding network between DR and DM molecules [31] and previous mutagenesis experiments supported the importance of $\alpha E 40$ [46]. Among the six teleost fish molecules which possess $\alpha \mathrm{W} 43$, half of them also possess both $\alpha \mathrm{K} 38$ and $\alpha \mathrm{E} 40$. Although the chicken and frog classical class II molecules possess different amino acids, the teleost fish DB group molecules of the synteny region "S1" or "S2" group and their closely 
related ones and also the classical-type DA group molecules possess these residues at the relevant positions in relatively high frequency (Additional file 10: Text S4). The coelacanth class II molecule also possesses $\alpha E 40$ [31]. In the hydrogen bonding network, $\alpha E 40$ of the DR molecule interacts with $\alpha \mathrm{R} 98$ of the DM molecule. The conservation of $\alpha \mathrm{K} 98$ can be observed in chicken [31] and frog DM molecules (Additional file 10: Text S4), and also often in the teleost fish $\mathrm{DB}$ group molecules and in some DA molecules (Additional file 10: Text S4). Therefore, various preconditions for the critical hydrogen bonding network between DM and DR molecules appear to have been already established in the common ancestor between teleost fish and tetrapods.

\section{A teleost fish aN125-containing class II molecule is not a DM-equivalent}

$\alpha \mathrm{N} 125$ is conserved in mammalian and chicken DM molecules as previously noted [31] and it is also conserved in frog DM molecules (Additional file 10: Text S4). $\alpha$ N125 is not specific to the DM molecule, but is also observed in many classical class II $\alpha$ chains of the tetrapod lineage including frog, coelacanth and human DQA-related mammalian molecules, as well as in some closely related nonclassical ones like human DOA (Additional file 10: TextS4). We could find, thus far, a single $\alpha \mathrm{N} 125$-containing class II sequence in teleost fish, namely, DDA of Atlantic salmon (Additional file 10: Text S4). In our previous paper, we reported that $D D A$ has little polymorphism based on available EST information. DDA does not possess two conserved peptide-binding asparagines ( $\alpha$ N62 and $\alpha$ N69) and it possesses a hydrophobic residue at the position of 69 as found in most of the other teleost fish DB and also tetrapod DM molecules. $D D A$ is predominantly expressed in spleen although in much lower amounts compared to the classical DAA [29] (Additional file 8: Text S2). Based on various observations described above, DDA could be classified as a non-classical class II molecule, although we do not know about DDB at present. When we conducted homology searches with Atlantic salmon DDA, we could not retrieve DDA-like sequences from genome sequence databases of other teleost fish species. Therefore, although DDA is a non-classical class II $\alpha$ chain possessing $\alpha \mathrm{N} 125$, it is not like DM that is highly conserved throughout tetrapod species.

\section{Novel teleost fish class II group DE}

In the present study, we identified a new teleost fish class II group called DE. As described above, $D E A$ and $D E B$ genes of Atlantic salmon do not show classical-type polymorphism and their expression levels are very low (Additional file 8: Text S2). Although the predicted Atlantic salmon DE molecule possesses both $\beta S 144$ and $\beta E 162$ for CD4-binding, it lacks $\alpha$ N62, $\beta$ H81 and $\beta$ N82 for peptide-binding. Based on these observations, we could exclude the Atlantic salmon DE molecule from the classical class II group. As also described in the previous section, the Atlantic salmon DE molecule shares several amino acid residues with the cartilaginous fish and tetrapod classical, and also tetrapod DM molecules (Figure 3, Additional file 10: Text S4). From the standpoint of the conservation of these residues, the $\mathrm{DE}$ molecule is closest to the DM molecules among all the known teleost fish class II molecules. All the available DEA sequences found in teleost fish and also in the primitive ray-finned fish possesses an aspartic acid residue at the position of 125 instead of an asparagine (Additional file 10: Text S4C). Intriguingly, an aspartic acid also can participate in a hydrogen-bond interaction with an indole ring nitrogen atom of a tryptophan like an asparagine e.g., [55].

The observation that the gar class II sequence of the conserved DE lineage possesses $\alpha \mathrm{D} 125$ and additionally another gar class II sequence (Additional file 10: Text S4C) possesses $\alpha \mathrm{N} 125$ suggests that $\alpha \mathrm{N} 125 / \mathrm{D} 125$ already appeared in the class II molecules of an ancestor of Osteichthyes, whereas available cartilaginous fish sequences possess a glycine at this position that appears ancestral to the MHC family [56] (Table 1, Additional file 10: Text S4C). It should be noted that a glycine cannot provide hydrogen-bond capacity necessary for the DM function. Together with the other residues already discussed above, $\alpha$ N125/D125 in ray-finned fish molecules further suggests the establishment of the preconditions for the critical hydrogen bonding network between DM and DR molecules in the common ancestor of teleost fish and tetrapods.

With the identification of DE genes in primitive rayfinned fish like the spotted gar, the coexistence of classical and non-classical class II lineages for a long evolutionary time was demonstrated in the present study (Figure 1). DE genes are found in some teleost fish, while they could not be identified in the genome databases and the EST databases of the other teleost fish. Thus, although DE molecules share some non-classical features with DM molecules, they seem to have quite different characteristics regarding stable inheritance.

\section{Teleost fish do not possess the DM system}

Without a complete coverage of teleost fish genomes, it is logically not possible to deny the existence of DMequivalent genes. With this limitation, we clarified that some non-classical class II genes exist in the teleost fish genomes whose protein products partially share some characteristics with the DM molecule. Actually there exist some teleost fish non-classical class II molecules that possess $\alpha \mathrm{N} 125$ or $\alpha \mathrm{D} 125$. Mainly based on their conservation profiles in the teleost fish genomes, they 
appear not to be DM-equivalents of teleost fish. In the middle of the $\beta 2$ domain, the tetrapod DM molecules have a unique insertion of several amino acid residues and near or in this region there are a few residues (e.g. $\beta E 47$ and $\beta L 51)$ influential for the DM/DR interaction supported by the mutation studies [47] and also by the structural study [31]. So far, teleost fish genes whose protein products possess these features have not been identified.

To obtain a clear conclusion about a possible DM system in teleost fish, it was necessary to investigate the other side of the DM mechanism, namely, the possession of the critical tryptophan residue in the classical class II molecules. Based on the analyses regarding $\alpha \mathrm{W} 43$ in the teleost fish classical class II molecules, we could conclude that teleost fish classical class II molecules do not possess $\alpha \mathrm{W} 43$ and therefore do not possess the DM system in which the interaction between $\alpha \mathrm{W} 43$ of the classical class II molecule and $\alpha \mathrm{N} 125$ of the DM molecule is critically important. Although we could observe some teleost fish class II molecules which possess $\alpha \mathrm{W} 43$, we could clearly classify them as non-classical. In contrast, coelacanth classical-type class II molecule has this tryptophan [31], and this might be consistent with the presence of the DM system in the primitive stage of the tetrapod lineage. Although available data are very limited, known classical polymorphic class II $\alpha$ chains of cartilaginous fish do not possess $\alpha \mathrm{W} 43$ (Figure 3, Additional file 10: Text S4A), e.g. the nurse shark molecule has an alanine at this position which does not have hydrogen-bonding capacity. Therefore, $\alpha$ W43 appears fixed in the classical class II $\alpha$ chain only from the level of coelacanth in the lobe-finned fish/tetrapod line [31] (Figure 3, Additional file 10: Text S4A). As the coelacanth class II molecule also possesses $\alpha \mathrm{N} 125$, both the important $\alpha \mathrm{W} 43$ and $\alpha \mathrm{N} 125$ prerequisites for the DM function may have been fixed at the early phase of the tetrapod lineage (Figure 1). All these observations support that the DM system has specifically evolved in the tetrapod lineage.

\section{Possible MHC class II peptide-presentation system of teleost fish without DM}

Studies on the teleost fish MHC class II peptidepresentation system have thus far been very limited. If we assume that the teleost fish invariant chain [21-23] possesses functions similar to those of mammals $[1,2]$, some basic issues need to be considered for the peptideloading pathway without the DM system. Those include the dissociation of CLIP-equivalent fragments from classical class II molecules and the stabilization of empty classical class II molecules during peptide-exchange reactions. First, as some mammalian classical MHC class II molecules bind CLIP with low affinity, the rapid dissociation of CLIP has been observed at an endosomal low $\mathrm{pH}[2,57]$. Therefore, if the binding of teleost fish
CLIP-equivalent fragments to classical class II molecules is not so strong, the fragments may dissociate without help of a DM-equivalent. Second, classical class II gene duplications can produce an evolutionary reservoir of nonclassical class II genes. Some classical MHC class II molecules possess intrinsic affinity for each other [58], and similar interactive forces may have been the evolutionary basis of the tetrapod DM molecules for establishing the specific interaction wtih classical class II molecules. Some teleost fish non-classical class II molecules may also have acquired stabilizing activity toward classical class II molecules in evolution. Thus, although mechanisms cannot be identical, there might be some overlap between the tetrapod DM and the teleost nonclassical class II functions in aiding classical class II molecules.

For distinct subgroups of teleost fish, we may find different strategies for the MHC class II peptide-loading. With four kinds of non-classical class II molecules, Atlantic salmon might have a peptide-loading system uniquely evolved with these molecules. Other than peptide presentation, some reports indicated that tetrapod MHC class II molecules can be involved in signaling pathways [1], and some teleost fish class II molecules may participate in similar or yet unknown functions.

\section{Conclusions}

The accelerated progress on whole genome sequence and also expressed sequence information of various species certainly is valuable to gain an evolutionary bird's-eye view of important biological systems. The observation that all the authentic polymorphic classical class II-type molecules of teleost fish do not possess the critical residue $\alpha W 43$ led us to conclude that teleost fish do not possess the DM system. The DM molecule appears to have acquired highly sophisticated and efficient mechanisms for peptide editing and stabilization of the classical class II molecules, ensuring its preservation throughout tetrapod evolution. As teleost fish comprise a significant part of the jawed vertebrates (more than $40 \%$ of all the species), the present study revealed that both DM-dependent and -independent systems are present as major fractions in the jawed vertebrates. Our study also suggests that preconditions necessary for the important hydrogen-bonding network in the DM system appeared in the common ancestor of teleost fish and tetrapods. Exploring the teleost fish class II peptide-loading system would constitute an important part for the comprehensive understanding of the MHC class II antigen-presentation systems in the jawed vertebrates. Future studies on the non-classical class II molecules of teleost fish should reveal whether they have functions to support the classical class II molecules like the DM molecules or other and yet unknown functions. 


\section{Methods}

\section{Data mining and bioinformatics}

A mixture of annotated and un-annotated MHC class II $\alpha$ and $\beta$ sequences were identified using Ensembl's Biomart and the GO term for class II (GO: 0042613) supplemented with various blastN and TblastN searches of Ensembl and NCBI databases using evolutionary diverged as well as species-specific sequences. For Atlantic salmon, we supplemented the six known Atlantic salmon MHC class II genes [29] with blastN and TblastN searches using available salmon genome sequences including and mostly from our ongoing genome sequencing project (in part available at either cGRASP (http://web.uvic.ca/grasp/) or NCBI (http://www.ncbi.nlm.nih.gov/)). Open reading frames were predicted using GenScan [59] and Fgenesh [60] and by comparison with known MHC sequences. Some small pseudogene remnants which did not contribute to evolutionary understanding were neglected.

\section{Expression pattern of Atlantic salmon MHC class II gene transcripts}

Expression of Atlantic salmon class II genes was estimated by transcriptome analysis and by gene-specific RT-PCR analysis. The transcriptome data, using tissues of a oneyear old salmon, comprised $>70.000$ non-redundant contigs and $>50$ million reads per tissue (Additional file 8 : Text S2 (Table TS2-2A)), and data on MHC class II expression agree fully with our previous RT-PCR analysis results on $D A A, D A B, D B A, D B B, D C A$ and $D D A$ [29] and our present RT-PCR analysis results on $D C B, D E A$ and $D E B$ for three adult salmon individuals (Additional file 8: Text S2-2B). For details of methods and results see Additional file 8: Text S2-2A and Additional file 8: Text S2-2B. The protocol for the ethics and use of the animals was in accordance with the Animal Care at the University of Victoria, and all animal experiments comply with the current laws of Norway.

\section{Phylogenetic analysis}

The alignments of the MHC class II sequences shown in Additional file 10: Text S4 were done manually, based on structural and evolutionary considerations. The evolutionary history of these manually aligned sequences was inferred using the Neighbor-Joining method [61] using MEGA5 software [62]. See Additional file 3: Figure S3 for details.

\section{Additional files}

Additional file 1: Figure S1. Phylogeny of relevant species.

Additional file 2: Figure S2. Teleost fish and gar MHC class II genomic regions.

Additional file 3: Figure S3. Phylogenetic trees of $\beta 1, a 2$ and $\beta 2$ domains.
Additional file 4: Table S1. MHC class II loci and their syntenic regions in selected teleosts.

Additional file 5: Table S2. MHC class II gene Ensembl IDs, Ensembl genomic locations, and number of matching cDNA reports in GenBank non-specific datasets, for zebrafish, stickleback, medaka, Fugu, Tetraodon, tilapia, and spotted gar.

Additional file 6: Table S3. Conservation pattern of residues which contribute to the hydrogen-bond network between classical MHC class II molecules and the backbone of peptide ligands.

Additional file 7: Text S1. Deduced MHC class II amino acid sequences of teleost fish and gar from Ensembl database and matching GenBank cDNA reports.

Additional file 8: Text S2. Atlantic salmon (Salmo salar) MHC class II sequences, transcripts and genomic regions.

Additional file 9: Text S3. Discussion of potential polymorphism of MHC class II genes in selected teleosts and comparison with previous studies.

Additional file 10: Text S4. MHC class II domain sequence alignments.

\section{Abbreviations}

CLIP: Class II-associated invariant chain peptide; MHC: Major histocompatibility complex; MIIC: MHC class II compartment; MYA Million years ago; TGD: Teleost-specific whole genome duplication; SGD: Salmonid-specific whole genome duplication.

\section{Competing interests}

The authors declare that they have no competing interests.

\section{Authors' contributions}

$J M D, U G, J L$ and BFK performed experiments and analysis. JMD, UG and KH wrote the paper. All authors read and approved the final manuscript.

\section{Acknowledgements}

This work was supported by JSPS KAKENHI Grant Number 22580213 and 25450310 (to J. M. D.), a Norwegian Research Council grant (Havbruk \# NFR 185217/S40) (to U. G.), an NSERC grant (to B. F. K.) and the MEXT-Supported Program for the Strategic Research Foundation at Private Universities, 2008-2012.

\section{Author details}

${ }^{1}$ Institute for Comprehensive Medical Science, Fujita Health University, Toyoake, Aichi 470-1192, Japan. ${ }^{2}$ Centre for Ecology and Evolutionary Synthesis, Department of Biosciences, University of Oslo, Postboks 1066 Blindern, Oslo 0316, Norway. ${ }^{3}$ Centre for Biomedical Research, Department of Biology, University of Victoria, PO Box 3020 STN CSC, Victoria, Canada.

Received: 21 August 2013 Accepted: 4 November 2013 Published: 26 November 2013

\section{References}

1. Neefjes J, Jongsma MLM, Paul P, Bakke O: Towards a systems understanding of MHC class I and MHC class II antigen presentation. Nature Rev Immunol 2011, 11:823-836

2. Schulze MS, Wucherpfennig KW: The mechanism of HLA-DM induced peptide exchange in the MHC class II antigen presentation pathway. Curr Opin Immunol 2012, 24:105-111.

3. Sloan VS, Cameron P, Porter G, Gammon M, Amaya M, Mellins E, Zaller DM: Mediation by HLA-DM of dissociation of peptides from HLA-DR. Nature 1995, 375:802-806.

4. Denzin LK, Cresswell P: HLA-DM induces CLIP dissociation from MHC classll a $\beta$ dimers and facilitates peptide loading. Cell 1995, 82:155-165.

5. Sherman MA, Weber DA, Jensen PE: DM enhances peptide binding to class II MHC by release of invariant chain-derived peptide. Immunity 1995, 3:197-205.

6. Weber DA, Evavold BD, Jensen PE: Enhanced dissociation of HLA-DRbound peptides in the presence of HLA-DM. Science 1996, 274:618-620. 
7. Morris P, Shaman J, Attaya M, Amaya M, Goodman S, Bergman C, Monaco J, Mellins E: An essential role for HLA-DM in antigen presentation by class II major histocompatibility molecules. Nature 1994, 368:551-554.

8. Fling SP, Arp B, Pious D: HLA-DMA and -DMB genes are both required for MHC class II/peptide complex formation in antigen-presenting cells. Nature 1994, 368:554-558.

9. Miyazaki T, Wolf P, Tourne S, Waltzinger C, Dierich A, Barois N, Ploegh H, Benoist C, Mathis D: Mice lacking H2-M complexes, enigmatic elements of the MHC class II peptide-loading pathway. Cell 1996, 84:531-541.

10. Fung-Leung W-P, Surh CD, Liljedahl M, Pang J, Leturca D, Peterson PA, Webb SR, Karlsson L: Antigen presentation and T cell development in H2-M-deficient Mice. Science 1996, 271:1278-1281

11. Mosyak L, Zaller DM, Wiley DC: The structure of HLA-DM, the peptide exchange catalyst that loads antigen onto class II MHC molecules during antigen presentation. Immunity 1998, 9:377-383.

12. Fremont DH, Crawford F, Marrack P, Hendrickson WA, Kappler J: Crystal structure of mouse H2-M. Immunity 1998, 9:385-393.

13. Kaufman J, Milne S, Göbel TW, Walker BA, Jacob JP, Auffray C, Zoorob R, Beck S: The chicken $B$ locus is a minimal essential major histocompatibility complex. Nature 1999, 401:923-925

14. Ohta Y, Goetz W, Hossain MZ, Nonaka M, Flajnik MF: Ancestral organization of the MHC revealed in the amphibian Xenopus. J Immunol 2006, 176:3674-3685.

15. Nelson JS: Fishes of the world. 4th edition. New York: Wiley; 2006.

16. Iwama G, Nakanishi T: The fish immune system. San Diego: Academic Press; 1996.

17. Hashimoto $K$, Nakanishi T, Kurosawa Y: Isolation of carp genes encoding major histocompatibility complex antigens. Proc Natl Acad Sci USA 1990, 87:6863-6867.

18. Sultmann H, Mayer WE, Figueroa F, O'hUigin C, Klein J: Zebrafish Mhc class II alpha chain-encoding genes: polymorphism, expression, and function. Immunogenetics 1993, 38:408-420.

19. Suetake H, Araki K, Suzuki Y: Cloning, expression, and characterization of fugu CD4, the first ectothermic animal CD4. Immunogenetics 2004, 56:368-374

20. Dijkstra JM, Somamoto T, Moore L, Hordvik I, Ototake M, Fischer U: Identification and characterization of a second CD4-like gene in teleost fish. Mol Immunol 2006, 43:410-419.

21. Yoder JA, Haire RN, Litman GW: Cloning of two zebrafish cDNAs that share domains with MHC class II-associated invariant chain. Immunogenetics 1999, 50:84-88.

22. Fujiki K, Smith CM, Liu L, Sundick RS, Dixon B: Alternate forms of MHC class II-associated invariant chain are not produced by alternative splicing in rainbow trout (Oncorhynchus mykiss) but are encoded by separate genes. Dev Comp Immunol 2003, 27:377-391.

23. Dijkstra JM, Kiryu I, Kollner B, Yoshiura Y, Ototake M: MHC class II invariant chain homologues in rainbow trout (Oncorhynchus mykiss). Fish Shellfish Immunol 2003, 15:91-105.

24. Bingulac-Popovic J, Figueroa F, Sato A, Talbot WS, Johnson SL, Gates M, Postlethwait JH, Klein J: Mapping of mhc class I and class II regions to different linkage groups in the zebrafish, Danio rerio. Immunogenetics 1997, 46:129-134.

25. Kuroda N, Figueroa F, O'HUigin C, Klein J: Evidence that the separation of Mhc class II from class I loci in the zebrafish, Danio rerio, occurred by translocation. Immunogenetics 2002, 54:418-430.

26. Stet RJ, Kruiswijk CP, Dixon B: Major histocompatibility lineages and immune gene function in teleost fishes: the road not taken. Crit Rev Immunol 2003, 23:441-471.

27. Star B, Nederbragt AJ, Jentoft S, Grimholt U, Malmstrøm M, Gregers TF, Rounge TB, Paulsen J, Solbakken MH, Sharma A, Wetten OF, Lanzén A, Winer R, Knight J, Vogel JH, Aken B, Andersen O, Lagesen K, ToomingKlunderud A, Edvardsen RB, Tina KG, Espelund M, Nepal C, Previti C, Karlsen BO, Moum T, Skage M, Berg PR, Gjøen T, Kuhl H, et al: The genome sequence of Atlantic cod reveals a unique immune system. Nature 2011, 477:207-210.

28. McConnell TJ, Godwin UB, Cuthbertson BJ: Expressed major histocompatibility complex class II loci in fishes. Immunol Rev 1998, 166:294-300.

29. Harstad H, Lukacs MF, Bakke HG, Grimholt U: Multiple expressed MHC class II loci in salmonids; details of one non-classical region in Atlantic salmon (Salmo salar). BMC Genomics 2008, 9:193.
30. Sato A, Dongak R, Hao L, Shintani S, Sato T: Organization of Mhc class II A and B genes in the tilapiine fish Oreochromis. Immunogenetics 2012, 64:679-690

31. Pos W, Sethi DK, Call MJ, Schulze MS, Anders AK, Pyrdol J, Wucherpfennig KW: Crystal structure of the HLA-DM-HLA-DR1 complex defines mechanisms for rapid peptide selection. Cell 2012, 151:1557-1568.

32. Palti $Y$, Rodriguez MF, Gahr SA, Hansen JD: Evolutionary history of the ABCB2 genomic region in teleosts. Dev Comp Immunol 2007, 31:483-498.

33. Ohashi K, Takizawa F, Tokumaru N, Nakayasu C, Toda H, Fischer U, Moritomo T, Hashimoto K, Nakanishi T, Dijkstra JM: A molecule in teleost fish, related with human MHC-encoded G6F, has a cytoplasmic tail with ITAM and marks the surface of thrombocytes and in some fishes also of erythrocytes. Immunogenetics 2010, 62:543-559.

34. Lukacs MF, Harstad H, Bakke HG, Beetz-Sargent M, McKinnel L, Lubieniecki KP, Koop BF, Grimholt U: Comprehensive analysis of MHC class I genes from the U-, S-, and Z-lineages in Atlantic salmon. BMC Genomics 2010, 11:154

35. Ohta Y, Okamura K, McKinney EC, Bartl S, Hashimoto K, Flajnik MF: Primitive synteny of vertebrate major histocompatibility complex class I and class II genes. Proc Natl Acad Sci USA 2000, 97:4712-4717.

36. Kulski JK, Shiina T, Anzai T, Kohara S, Inoko H: Comparative genomic analysis of the MHC: the evolution of class I duplication blocks, diversity and complexity from shark to man. Immunol Rev 2002, 190:95-122.

37. Cadavid LF, Mejia BE, Watkins DI: MHC class I genes in a New World primate, the cotton-top tamarin (Saguinus oedipus), have evolved by an active process of loci turnover. Immunogenetics 1999, 49:196-205.

38. Fremont DH, Monnaie D, Nelson CA, Hendrickson WA, Unanue ER: Crystal structure of $\mathrm{I}-\mathrm{A}^{\mathrm{k}}$ in complex with a dominant epitope of lysozyme. Immunity 1998, 8:305-317.

39. Painter $C A$, Stern $\sqcup$ : Conformational variation in structures of classical and non-classical MHCII proteins and functional implications. Immunol Rev 2012, 250:144-157.

40. Zhou Z, Callaway KA, Weber DA, Jensen PE: Cutting Edge: HLA-DM functions through a mechanism that does not require specific conserved hydrogen bonds in class II MHC-peptide complexes. J Immunol 2009, 183:4187-4191.

41. Salomonsen J, Marston D, Avila D, Bumstead N, Johansson B, Juul-Madsen $H$, Olesen GD, Riegert P, Skjødt K, Vainio O, Wiles MV, Kaufman J: The properties of the single chicken MHC classical class II a chain $(B-L A)$ gene indicate an ancient origin for the DR/E-like isotype of class II molecules. Immunogenetics 2003, 55:605-614.

42. Wang XX, Li Y, Yin Y, Mo M, Wang Q, Gao W, Wang L, Mariuzza RA: Affinity maturation of human CD4 by yeast surface display and crystal structure of a CD4-HLA-DR1 complex. Proc Natl Acad Sci USA 2011, 108:15960-15965.

43. Lindstedt R, Liljedahl M, Peleraux A, Peterson PA, Karlsson L: The MHC class II molecule H2-M is targeted to an endosomal compartment by a tyrosine-based targeting motif. Immunity 1995, 3:561-572.

44. Marks MS, Roche PA, van Donselaar E, Woodruff L, Peters PJ, Bonifacino JS: A lysosomal targeting signal in the cytoplasmic tail of the $\beta$ chain directs HLA-DM to MHC class II compartments. J Cell Biol 1995, 131:351-369.

45. Hughes AL, Nei M: Evolution of the major histocompatibility complex: independent origin of nonclassical class I genes in different groups of mammals. Mol Biol Evol 1989, 6:559-579.

46. Doebele RC, Busch R, Scott HM, Pashine A, Mellins ED: Determination of the HLA-DM interaction site on HLA-DR molecules. Immunity 2000, 13:517-527.

47. Pashine A, Busch R, Belmares MP, Munning JN, Doebele RC, Buckingham M, Nolan GP, Mellins ED: Interaction of HLA-DR with an acidic face of HLA-DM disrupts sequence-dependent interactions with peptides. Immunity 2003, 19:183-192.

48. Anders A-K, Call MJ, Schulze M-S ED, Fowler KD, Schubert DA, Seth NP Sundberg EJ, Wucherpfennig KW: HLA-DM captures partially empty HLA-DR molecules for catalyzed removal of peptide. Nat Immunol 2011, 12:54-61.

49. Painter CA, Negroni MP, Kellersberger KA, Zavala-Ruiz Z, Evans JE, Stern LJ: Conformational lability in the class II MHC $3_{10}$ helix and adjacent extended strand dictate HLA-DM susceptibility and peptide exchange. Proc Natl Acad Sci USA 2011, 108:19329-19334.

50. Stratikos E, Mosyak L, Zaller DM, Wiley DC: Identification of the lateral interaction surfaces of human histocompatibility leukocyte antigen (HLA)-DM with HLA-DR1 by formation of tethered complexes that present enhanced HLA-DM catalysis. J Exp Med 2002, 196:173-183. 
51. Stern $L$, Brown JH, Jardetzky TS, Gorga JC, Urban RG, Strominger JL, Wiley DC Crystal structure of the human class II MHC protein HLA-DR1 complexed with an influenza virus peptide. Nature 1994, 368:215-221.

52. Guce Al, Mortimer SE, Yoon T, Painter CA, Jiang W, Mellins ED, Stern L: HLA-DO acts as a substrate mimic to inhibit HLA-DM by a competitive mechanism. Nature Struct Mol Biol 2013, 20:90-98.

53. Stet RJM, De Vries B, Mudde K, Hermsen T, Van Heerwaarden J, Shum BP, Grimholt U: Unique haplotypes of co-segregating major histocompatibility class II A and class II B alleles in Atlantic salmon (Salmo salar) give rise to diverse class II genotypes. Immunogenetics 2002, 54:320-331.

54. Murphy K: Janeway's Immunobiology. 8th edition. New York: Garland Science; 2012.

55. Cordero-Morales JF, Jogini V, Chakrapani S, Perozo E: A multipoint Hydrogen-bond network underlying KcsA C-type inactivation. Biophys J 2011, 100:2387-2393.

56. Dijkstra JM, Katagiri T, Hosomichi K, Yanagiya K, Inoko H, Ototake M, Aoki T, Hashimoto K, Shiina T: A third broad lineage of major histocompatibility complex (MHC) class I in teleost fish; MHC class II linkage and processed genes. Immunogenetics 2007, 59:305-321.

57. Hausmann DHF, Yu B, Hausmann S, Wucherpfennig KW: pH dependent peptide binding properties of the type I diabetes-associated I- $\mathrm{A}^{\mathrm{g}}$ molecule: rapid release of CLIP at an endosomal pH. J Exp Med 1999, 189:1723-1733.

58. Schafer PH, Pierce SK, Jardetzky TS: The structure of MHC class II: a role for dimer of dimers. Seminars Immunol 1995, 7:389-398.

59. Burge C, Karlin S: Prediction of complete gene structures in human genomic DNA. J Mol Biol 1997, 268:78-94.

60. Salamov AA, Solovyev W: Ab initio gene finding in Drosophila genomic DNA. Genome Res 2000, 10:516-522.

61. Saitou N, Nei M: The neighbor-joining method: a new method for reconstucting phylogenetic trees. Mol Biol Evol 1987, 4:406-425.

62. Tamura K, Peterson D, Peterson N, Stecher G, Nei M, Kumar S: MEGA5: molecular evolutionary genetics analysis using maximum likelihood, evolutionary distance, and maximum parsimony methods. Mol Biol Evol 2011, 28:2731-2739.

doi:10.1186/1471-2148-13-260

Cite this article as: Dijkstra et al: Comprehensive analysis of MHC class II genes in teleost fish genomes reveals dispensability of the peptideloading DM system in a large part of vertebrates. BMC Evolutionary Biology 2013 13:260.

\section{Submit your next manuscript to BioMed Central and take full advantage of:}

- Convenient online submission

- Thorough peer review

- No space constraints or color figure charges

- Immediate publication on acceptance

- Inclusion in PubMed, CAS, Scopus and Google Scholar

- Research which is freely available for redistribution 